\title{
Timelike vs Spacelike DVCS from JLab, Compass to Ultraperipheral Collisions and AFTER@LHC ${ }^{\S}$
}

\author{
H. Moutarde ${ }^{1}$, B. Pire ${ }^{2}$, F. Sabatié ${ }^{1}$, L. Szymanowski ${ }^{* 3}$ and J. Wagner ${ }^{3}$ \\ ${ }^{I}$ Irfu-SPhN, CEA, Saclay, France \\ ${ }^{2}$ CPhT, École Polytechnique, CNRS, 91128 Palaiseau, France \\ ${ }^{3}$ National Centre for Nuclear Research (NCBJ), Warsaw, Poland
}

\begin{abstract}
Timelike and spacelike virtual Compton scattering in the generalized Bjorken scaling regime are complementary tools to access generalized parton distributions. We stress that the gluonic contributions are by no means negligible, even in the medium energy range which will be studied intensely at JLab12 and in the COMPASS-II experiment at CERN. Ultraperipheral collisions with proton or ion beams may also be used at RHIC and at collider or fixed target experiments at LHC.
\end{abstract}

Keywords: Deeply virtual Compton scattering, Generalized Parton Distributions, LHC, timelike Compton scattering, Ultraperipheral collisions.

\section{INTRODUCTION}

In the collinear factorization framework the scattering amplitudes for exclusive processes such as deeply virtual Compton scattering (DVCS) [1]

$\gamma^{*}\left(q_{\text {in }}\right) N(P) \rightarrow \gamma\left(q_{\text {out }}\right) N^{\prime}\left(P^{\prime}=P+\Delta\right)$,

$q_{\text {in }}^{2}=-Q^{2}<0, \quad q_{\text {out }}^{2}=0$,

and its crossed reaction, timelike Compton scattering (TCS) [2]

$\gamma\left(q_{\text {in }}\right) N(P) \rightarrow \gamma^{*}\left(q_{\text {out }}\right) N^{\prime}\left(P^{\prime}=P+\Delta\right)$,

$q_{\text {in }}^{2}=0, \quad q_{\text {out }}^{2}=Q^{2}>0$,

have been shown to factorize in specific kinematical regions, provided a large scale controls the separation of short distance dominated partonic subprocesses and long distance hadronic matrix elements, the generalized quark and gluon distributions (GPDs) [3] which encode much information on the partonic content of nucleons. After factorization, the DVCS (and similarly TCS) amplitudes are written in terms of Compton form factors (CFF) $\mathcal{H}, \mathcal{E}$ and $\widetilde{\mathcal{H}}, \widetilde{\mathcal{E}}$, as:

$$
\begin{aligned}
& \mathcal{A}^{\mu v}(\xi, t)=-e^{2} \frac{1}{\left(P+P^{\prime}\right)^{+}} \bar{u}\left(P^{\prime}\right)\left[g _ { T } ^ { \mu v } \left(\mathcal{H}(\xi, t) \gamma^{+}+\right.\right. \\
& \left.\left.\mathcal{E}(\xi, t) \frac{i \sigma^{+\rho} \Delta_{\rho}}{2 M}\right)+i \varepsilon_{T}^{\mu v}\left(\widetilde{\mathcal{H}}(\xi, t) \gamma^{+} \gamma_{5}+\widetilde{\mathcal{E}}(\xi, t) \frac{\Delta^{+} \gamma_{5}}{2 M}\right)\right] u(P),
\end{aligned}
$$

with the CFFs defined as:

*Address correspondence to this author at the National Centre for Nuclear Research (NCBJ), Warsaw, Poland;

E-mail: Lech.Szymanowski@fuw.edu.pl

${ }^{\S}$ Presented at the Low x workshop, May 30 - June 4 2013, Rehovot and Eilat, Israel.

$$
\begin{aligned}
& \mathcal{H}(\xi, t)=+\int_{-1}^{1} d x\left(\sum_{q} T^{q}(x, \xi) H^{q}(x, \xi, t)+T^{g}(x, \xi) H^{g}(x, \xi, t)\right), \\
& \widetilde{\mathcal{H}}(\xi, t)=+\int_{-1}^{1} d x\left(\sum_{q} T^{q}(x, \xi) H^{q}(x, \xi, t)+T^{g}(x, \xi) H^{g}(x, \xi, t)\right), \\
& (\xi, t)=-\int_{-1}^{1} d x\left(\sum_{q} \widetilde{T}^{q}(x, \xi) \widetilde{T}^{q}(x, \xi, t)+\widetilde{T}^{g}(x, \xi) \widetilde{T}^{g}(x, \xi, t)\right) .
\end{aligned}
$$

We report in Sect. 2 on a recent NLO analysis $[4,5]$ of DVCS and TCS amplitudes, and make a few remarks on the study of TCS in ultraperipheral collisions at hadron colliders (Sect. 3) and at fixed target experiments at LHC (Sect. 4).

\section{ON THE IMPORTANCE OF GLUONIC CONTRIBUTIONS}

\subsection{Gluonic Effects to Compton form Factors}

TCS and DVCS amplitudes are identical (up to a complex conjugation) at lowest order in $\alpha_{S}$ but differ at next to leading order, in particular because of the quite different analytic structure of the scattering amplitudes of these reactions. Indeed, the production of a timelike photon enables the production of intermediate states in some channels which are kinematically forbidden in the DVCS case. This opens the way to new absorptive parts of the amplitude. To estimate Compton Form Factors (CFF), we use the NLO calculations of the coefficient functions which have been calculated in the DVCS case in the early days of GPD studies and more recently for the TCS case [4], the two results being simply related thanks to the analyticity (in $Q^{2}$ ) properties of the amplitude [6]:

${ }^{T C S} T(x, \eta)= \pm\left({ }^{D V C S} T(x, \xi=\eta)+i \pi C_{\text {coll }}(x, \xi=\eta)\right)^{*}$, 

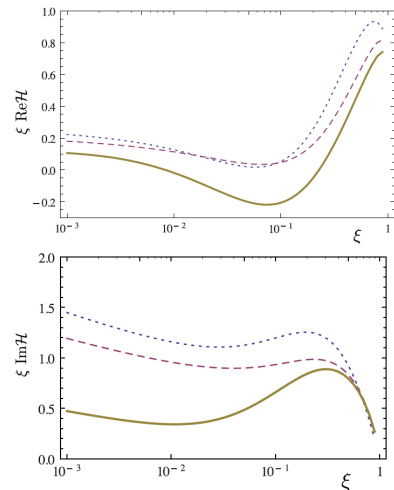
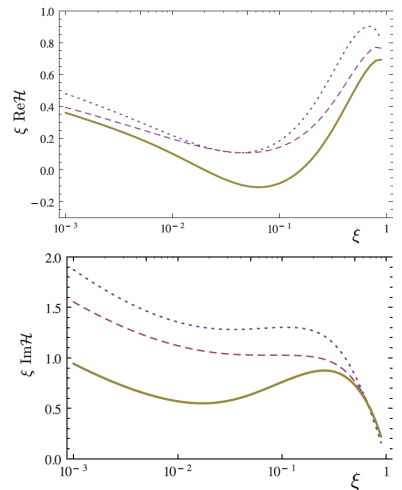
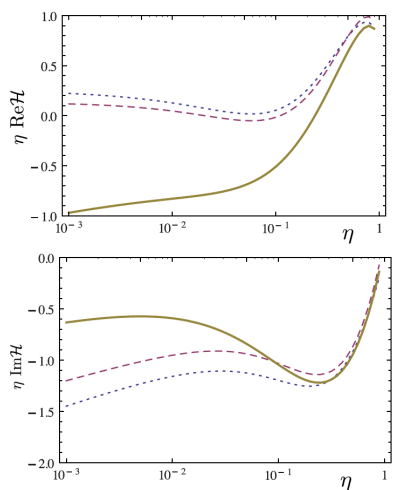
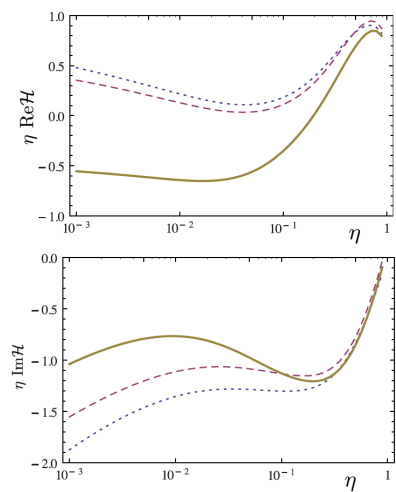

Fig. (1). The real (upper panels) and imaginary (lower pannels) parts of the spacelike $\mathcal{H}(\xi)\left(1^{\text {st }}\right.$ and $2^{\text {nd }}$ columns) and timelike $\mathcal{H}(\eta)\left(3^{\text {rd }}\right.$ and $4^{\text {th }}$ columns) Compton Form Factor multiplied by $\xi$ (or $\eta$ ), in the double distribution model based on GK ( $1^{\text {st }}$ and $3^{\text {rd }}$ columns) and $\operatorname{MSTW08}\left(2^{\text {nd }}\right.$ and $4^{\text {th }}$ columns) parametrizations, for $\mu_{F}^{2}=Q^{2}=4 G e V^{2}$ and $t=-0.1 G e V^{2}$. In all plots, the LO result is shown as the dotted line, the full NLO result by the solid line and the NLO result without the gluonic contribution as the dashed line.
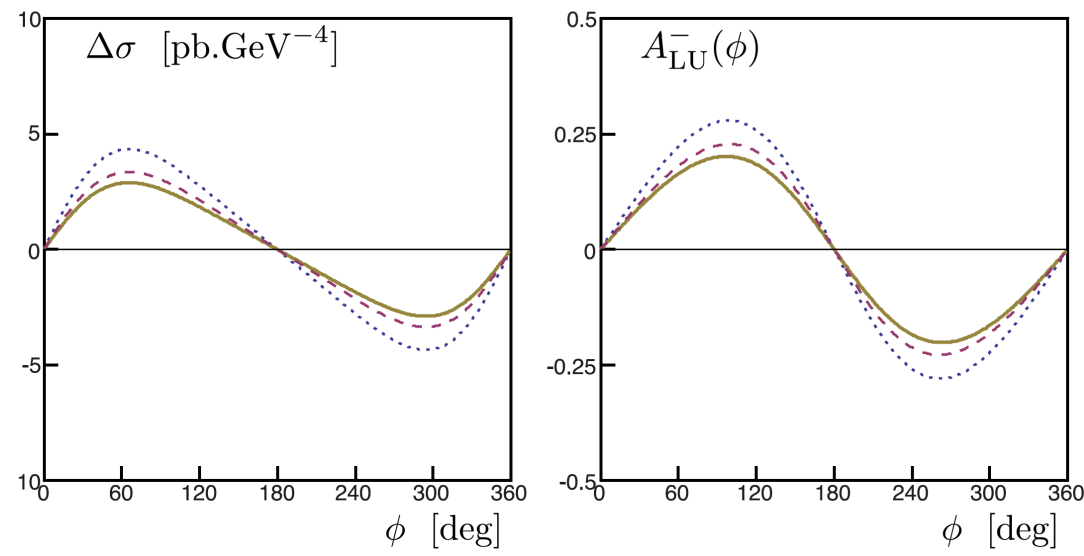

Fig. (2). The difference of DVCS cross sections for opposite lepton helicities in $\mathrm{pb} / \mathrm{GeV}^{4}$ (left) and the corresponding asymmetry (right), as a function of $\phi$ for $E_{e}=11 \mathrm{GeV}, \mu_{F}^{2}=Q^{2}=4 \mathrm{GeV}^{2}$ and $t=-0.2 \mathrm{GeV}^{2}$. The GPD $H(x, \xi, t)$ is parametrized by the GK model. The contributions from other GPDs are not included.

where $+(-)$ sign corresponds to vector (axial) case.

Using two GPD models based on Double Distributions (DDs), as discussed in detail in [5]: the Goloskokov-Kroll (GK) model [7] and a model based on the MSTW08 PDF parametrization [8], we get the results shown in Fig. (1) for the real and imaginary parts of the spacelike and timelike dominant $\mathrm{CFF} \mathcal{H}(\xi, t)$ and $\mathcal{H}(\eta, t)$. Comparing dashed and solid lines in the upper panels, one sees that gluonic contributions are so important that they even change the sign of the real part of the CFF, and are dominant for almost all values of the skewness parameter. A moderate result arises from a similar comparison of the lower panels; the gluonic contribution to the imaginary part of the CFF remains sizeable for values of the skewness parameter up to 0.3 .

\subsection{Gluonic Effects to DVCS Observables}

The effects of NLO contributions to some of the DVCS observables at moderate energies are exemplified in Figs. (2, 3) which show specific observables to be measured at JLab and COMPASS. The difference between the dotted and solid lines demonstrates that NLO contributions are important, whereas the difference between the dashed and solid lines shows that gluon contributions should not be forgotten even at low energy when a precise data set is analyzed.

\section{TCS IN ULTRAPERIPHERAL COLLISIONS}

Timelike Compton scattering in ultraperipheral collisions at hadron colliders opens a new way to measure generalized parton distributions, in particular for very small values of the skewness parameter.

We estimated [9] the different contributions to the lepton pair cross section for ultraperipheral collisions at the LHC. Since the cross sections decrease rapidly with $Q^{2}$, we are interested in the kinematics of moderate $Q^{2}$, say a few $\mathrm{GeV}^{2}$, and large energy, thus very small values of $\eta$. Note however that for a given proton energy the photon flux is higher at smaller photon energy.

The Bethe-Heitler amplitude grows much when small $\theta$ angles are allowed. In the following we will use the limits $[\pi / 4,3 \pi / 4]$ where the Bethe-Heitler cross section is sufficiently big but does not dominate too much over the Compton process. The Bethe-Heitler cross section integrated over $\theta \in[\pi / 4,3 \pi / 4]$, 

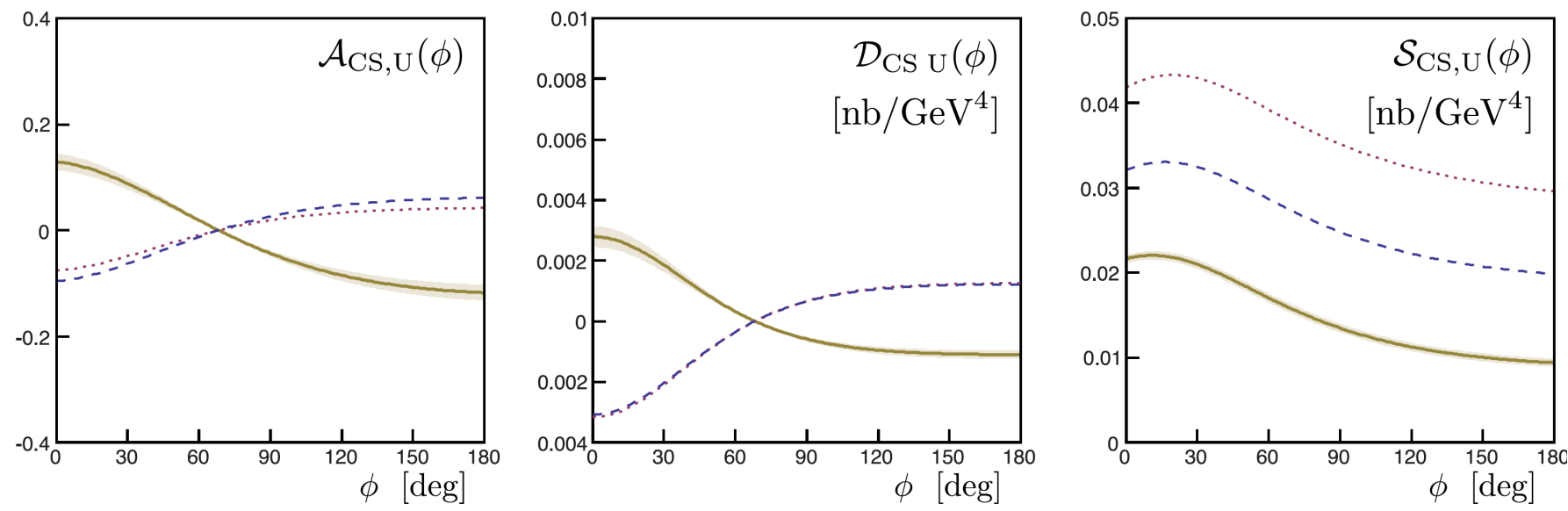

Fig. (3). The DVCS observables for the COMPASS experiment, from left to right, mixed charge-spin asymmetry, mixed charge-spin difference and mixed charge-spin sum (in $\mathrm{nb} / \mathrm{GeV}^{4}$ ). The kinematical point is chosen as $\xi=0.05, Q^{2}=4 \mathrm{GeV}^{2}, t=-0.2 \mathrm{GeV}$. The $\mathrm{GPD}$ $H(x, \xi, t)$ is parametrized in the double distribution model based on the MSTW08 parametrization. The contributions from other GPDs are not included.

$\phi \in[0,2 \pi], Q^{2} \in[4.5,5.5] \mathrm{GeV}^{2},|t| \in[0.05,0.25] \mathrm{GeV}^{2}$, as a function of $\gamma p$ energy squared $s$ is in the limit of large $s$ constant and equals $28.4 \mathrm{pb}$.

Since the amplitudes for the Compton and Bethe-Heitler processes transform with opposite signs under reversal of the lepton charge, the interference term between TCS and BH is odd under exchange of the $\ell^{+}$and $\ell^{-}$momenta. It is thus possible to project out the interference term through a clever use of the angular distribution of the lepton pair. The interference part of the cross-section for $\gamma p \rightarrow \ell^{+} \ell^{-} p$ with unpolarized protons and photons has a characteristic $(\theta, \phi)$ dependence given by (see details in [9]).

$\frac{d \sigma_{I N T}}{d Q^{2} d t d \cos \theta d \varphi}=-\frac{\alpha_{e m}^{3}}{4 \pi s^{2}} \frac{1}{-t} \frac{M}{Q} \frac{1}{\tau \sqrt{1-\tau}} \cos \varphi \frac{1+\cos ^{2} \theta}{\sin \theta} \operatorname{ReM}$,

with
$M=\frac{2 \sqrt{t_{0}-t}}{M} \frac{1-\eta}{1+\eta}\left[F_{1} H_{1}-\eta\left(F_{1}+F_{2}\right) H_{1}-\frac{t}{4 M^{2}} F_{2} E_{1}\right]$,

where $\tau=Q^{2} / s,-t_{0}=4 \eta^{2} M^{2} /\left(1-\eta^{2}\right)$ and $H, H, E$ are Compton form factors. With the integration limits symmetric about $\theta=\pi / 2$ the interference term changes sign under $\phi \rightarrow \pi+\phi$ due to charge conjugation, whereas the TCS and $\mathrm{BH}$ cross sections do not. One may thus extract the Compton amplitude through a study of $\int_{0}^{2 \pi} d \varphi \cos \varphi \frac{d \sigma}{d \varphi}$.

The lepton pair production differential cross sections (solid lines) for $t=-0.2 \mathrm{GeV}^{2}, Q^{2}=5 \mathrm{GeV}^{2}$ integrated over $\theta=[\pi / 4,3 \pi 4]$, as a function of $\phi$, for $s=10^{7} \mathrm{GeV}^{2}$ (a) $s=10^{5} \mathrm{GeV}^{2}$ (b) with $\mu_{F}^{2}=5 \mathrm{GeV}^{2}$. We also display the Compton (dotted), Bethe-Heitler (dash-dotted) and Interference (dashed) contributions. (a)

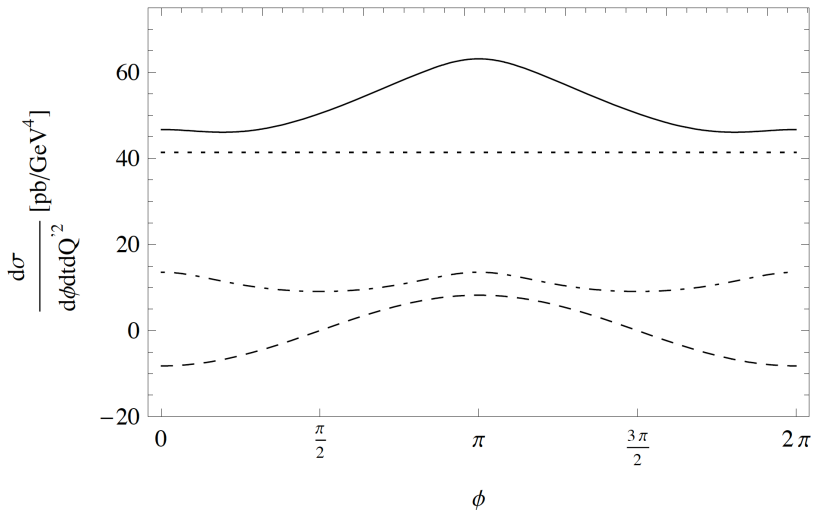

(b)

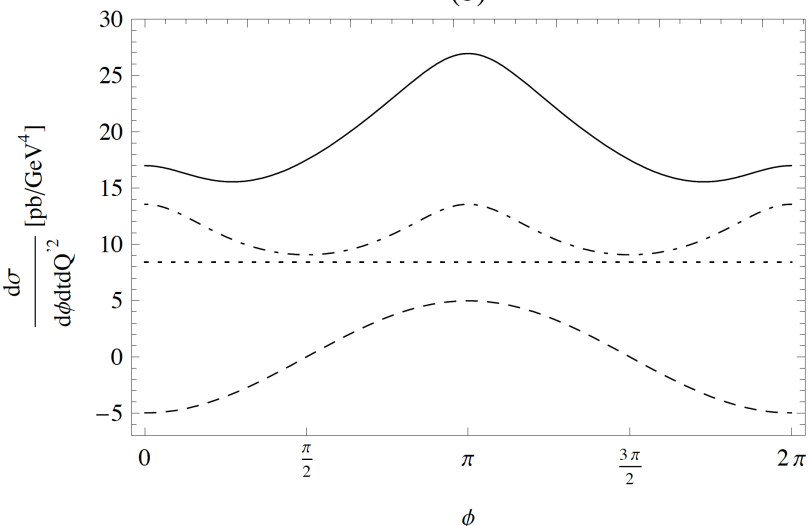

Fig. (4). The lepton pair production differential cross sections (solid lines) for $t=-0.2 \mathrm{GeV}^{2}, Q^{2}=5 \mathrm{GeV}$ integrated over $\theta=[\pi / 4 ; 3 \pi / 4]$, as a function of $\phi$, for $s=10^{7} \mathrm{GeV}^{2}$ (a) $s=10^{5} \mathrm{GeV}^{2}$ (b) with $\mu_{F}^{2}=5 \mathrm{GeV}^{2}$. We also display the Compton (dotted), Bethe-Heitler (dash-dotted) and Interference (dashed) contributions. 


\section{$\mathrm{LO} \mathrm{CF}$}

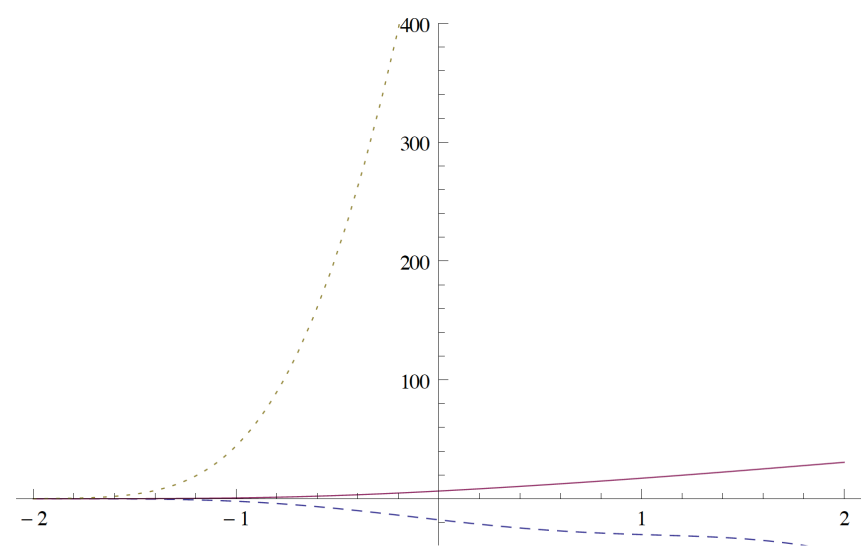

NLO CF

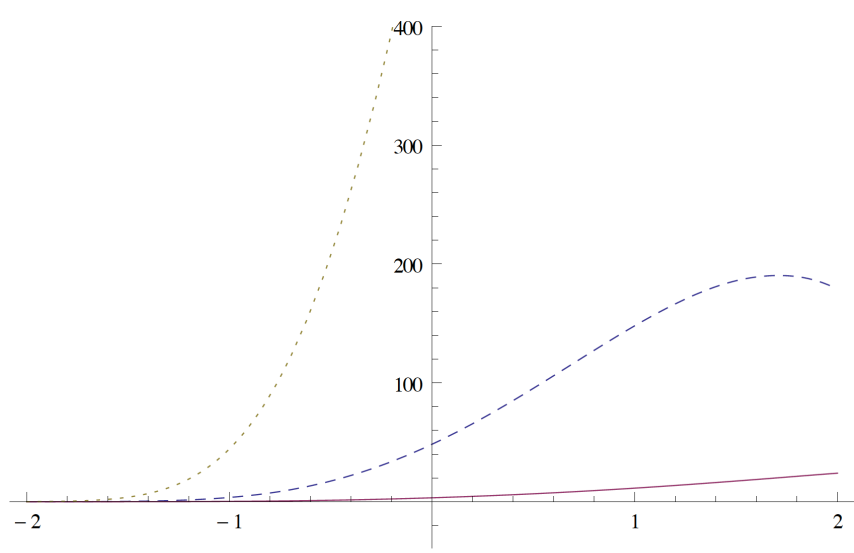

Fig. (5). The Bethe-Heitler(dotted), TCS(solid) and Interference(dashed) contributions to $\frac{d \sigma}{d Q^{2} d t d y d \varphi}$ in $p b / G e V^{4}$ as a function of y (in CMS) for $Q^{2}=4 G e V^{2}, t=-0.1 G e V^{2}, \varphi=0$ for the proton beam and $P b$ target case.

\section{$\mathrm{LO} \mathrm{CF}$}

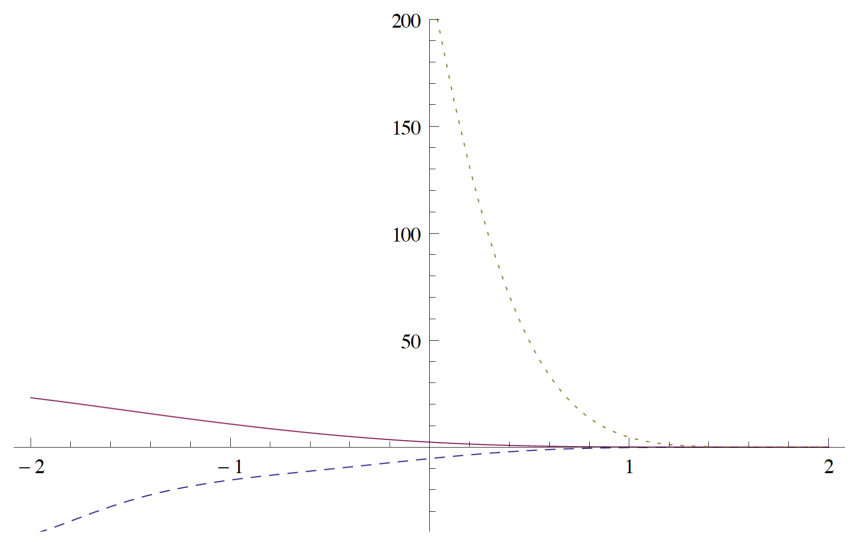

\section{NLO CF}

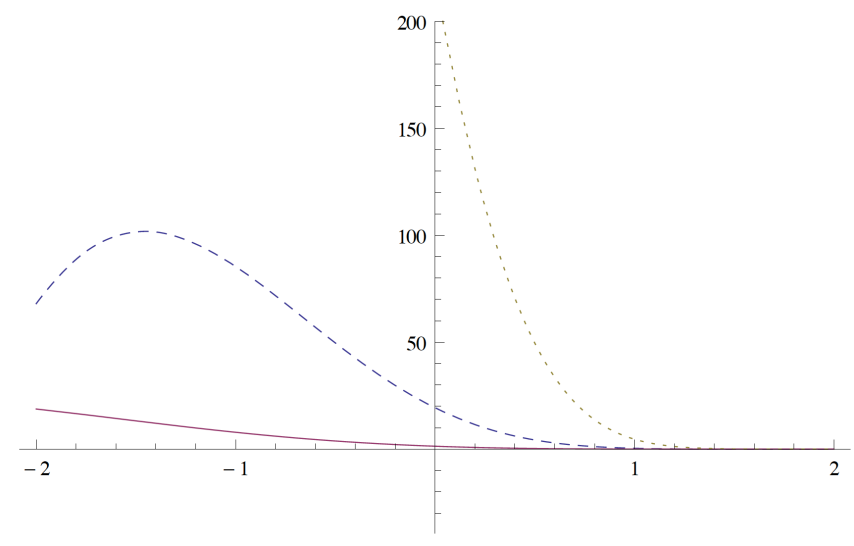

Fig. (6). The Bethe-Heitler(dotted), TCS(solid) and Interference(dashed) contributions to $\frac{d \sigma}{d Q^{2} d t d y d \varphi}$ in $p b / G e V^{4}$ for $\mathrm{BH}(\mathrm{dotted})$, TCS(solid), Interference(dashed) as a function of y (in CMS) for $Q^{2}=4 G e V^{2}, t=-0.1 G e V^{2}, \varphi=0$, for the $P b$ beam and proton target case.

In Fig. (4) we show the interference contribution to the cross section in comparison to the Bethe-Heitler and Compton processes, for various values of photon proton energy squared $s=10^{7} \mathrm{GeV}^{2}, 10^{5} \mathrm{GeV}^{2}$. We observe that for larger energies the Compton process dominates, whereas for $s=10^{5} \mathrm{GeV}^{2}$ all contributions are comparable.

\section{ULTRAPERIPHERAL COLLISIONS IN A HIGH- ENERGY FIXED-TARGET EXPERIMENT AFTER@ LHC}

The main idea of the multi-purpose project AFTER@LHC is to extract the halo of the LHC proton or ion beams by means of a bent-cristal and to use it as a beam in the fixed-target experiments [10]. The extracted beams will have a sufficiently high energy to produce on fixed target in ultraperipheral scattering a lepton pair with high invariant mass or heavy mesons. In these experiments a nucleus projectile or a nucleus target is treated as a highenergy photon source which allows study of photon-hadron collisions. Figs. (5 and $\mathbf{6}$, respectively) shows preliminary estimates of the Bethe-Heitler, TCS, and Interference contributions to the cross section, as functions of the CMS rapidity $y$, after integration over $\theta \in(\pi / 4,3 \pi / 4)$, in the region where the interference contribution is best seen, for the collision of a proton beam with a $\mathrm{Pb}$ target (and of a $\mathrm{Pb}$ beam with a proton target respectively). The double distribution model of GPDs based on MSTW08 parametrization is used.

\section{SUMMARY AND OUTLOOK}

We did not discuss here the rich phenomenology of DVCS and TCS processes which electron-ion colliders [11] 
will allow to study. Neither did we comment on recent progresses in higher twist contributions [12] nor on the effect of resummation of higher order QCD corrections [13]. The physics of generalized parton distributions is definitely a domain of work in progress, both on the theory and on the experimental side.

\section{CONFLICT OF INTEREST}

The authors confirm that this article content has no conflicts of interest.

\section{ACKNOWLEDGMENTS}

This work is supported by the Polish Grant NCN No DEC-2011/01/B/ST2/03915, the French-Polish collaboration agreement Polonium, the ANR project "Partons", the COPIN-IN2P3 Agreement and the Joint Research Activity "Study of Strongly Interacting Matter" (HadronPhysics3, Grant Agreement no. 283286) under the $7^{\text {th }}$ Framework Programme of the European Community.

\section{REFERENCES}

(a). Müller D, Robaschik D, Geyer B, Dittes FM, Hořejši J. Wave functions, evolution equations and evolution kernels from light-ray operators of QCD. Fortsch Phys 1994; 42: 101-41. (b). Radyushkin AV. Scaling limit of deeply virtual compton scattering. Phys Lett 1996; B380: 417-25. (c). Ji XD, Osborne J. One loop corrections and all order factorization in deeply virtual compton scattering. Phys Rev 1998; D58: 094018. (d). Collins J, Freund A. Proof of Factorization for Deeply Virtual Compton Scattering in QCD. Phys Rev 1999; D59: 074009.

[2] Berger ER, Diehl M, Pire B. Timelike Compton scattering: Exclusive photoproduction of lepton pairs. Eur Phys J 2002; C23: 675-89.

[3] (a). Goeke K, Polyakov MV, Vanderhaeghen M. Hard exclusive reactions and the structure of hadrons. Prog Part Nucl Phys 2001; 47: 401-515. (b). Diehl M. Generalized parton distributions. Phys Rep 2003; 388: 41-227. (c). Belitsky AV, Radyushkin AV.
Unraveling hadron structure with generalized parton distributions. Phys Rep 2005; 418: 1-378.

[4] (a). Pire B, Szymanowski L, Wagner J. NLO corrections to timelike, spacelike and double deeply virtual Compton scattering. Phys Rev 2011; D83: 034009. (b). Pire B, Szymanowski L, Wagner J. On timelike compton scattering at medium and high energies. Few Body Syst 2012; 53: 125-7.

[5] Moutarde H, Pire B, Sabatie F, Szymanowski L, Wagner J. On timelike and spacelike deeply virtual Compton scattering at next to leading order. Phys Rev D 2013; 87: 054029.

[6] Mueller D, Pire B, Szymanowski L, Wagner J. Timelike and spacelike hard exclusive reactions. Phys Rev D 2012; 86: 031502.

[7] Goloskokov SV, Kroll P. The role of the quark and gluon GPDs in hard vector-meson electroproduction. Eur Phys J 2008; C53: 36784 .

[8] Martin AD, Stirling WJ, Thorne RS, Watt G. Parton distributions for the LHC. Eur Phys J 2009; C63: 189-285.

[9] (a). Pire B, Szymanowski L, Wagner J. Can one measure timelike Compton scattering at LHC? Phys Rev D 2009; 79: 014010. (b). Pire B, Szymanowski L, Wagner J. Timelike compton scattering at LHC. Acta Phys Polon Supp 2009; 2: 373-8. (c). Pire B, Szymanowski L, Wagner J. Exclusive photoproduction of lepton pairs at LHC. Nucl Phys Proc Suppl 2008; 232: 179-80.

[10] (a). Brodsky SJ, Fleuret F, Hadjidakis C, Lansberg JP. Physics opportunities of a fixed-target experiment using LHC beams. Phys Rep 2013; 522: 239-55. (b). Lansberg JP, Arnaldi R, Brodsky SJ, et al.AFTER@LHC: a precision machine to study the interface between particle and nuclear physics. EPJ Web Conf 2014; 66: 11023.

[11] (a). Boer D, Diehl M, Milner R, et al. Gluons and the quark sea at high energies: distributions, polarization, tomography [arXiv: 1108 1713 [nucl-th]]. (b). Abelleira Fernandez JL, Adolphsen C, Akay AN, et al. [LHeC Study Group Collaboration]. A large hadron electron collider at CERN: Report on the physics and design concepts for machine and detector. J Phys G 2012; 39: 075001.

[12] (a). Braun VM, Manashov AN, Pirnay B. Finite-t and target-mass corrections to deeply virtual compton scattering. Phys Rev Lett 2012; 109: 242001. (b). Pire B, Szymanowski L, Wallon S. Chiralodd pion generalized parton distributions beyond leading twist. [arXiv:1309.0083 [hep-ph]].

[13] Altinoluk T, Pire B, Szymanowski L, Wallon S. Resumming soft and collinear contributions in deeply virtual Compton scattering. J High Energy Phys 2012; 1210: 049. 